\title{
INVESTIGATIVE UROLOGY
}

\author{
Pelvic floor exercises for erectile dysfunction \\ Dorey G, Speakman MJ, Feneley RC, Swinkels A, Dunn CD \\ The Somerset Nuffield Hospital, Taunton, UK \\ BJU Int. 2005; 96: 595-7
}

Objective: To examine the role of pelvic floor exercises as a way of restoring erectile function in men with erectile dysfunction.

Patients and Methods: In all, 55 men aged $>20$ years who had experienced erectile dysfunction for $\geq 6$ months were recruited for a randomized controlled study with a cross-over arm. The men were treated with either pelvic floor muscle exercises (taught by a physiotherapist) with biofeedback and lifestyle changes (intervention group) or they were advised on lifestyle changes only (control group). Control patients who did not respond after 3 months were treated with the intervention. All men were given home exercises for a further 3 months. Outcomes were measured using the International Index of Erectile Function (IIEF), anal pressure measurements and independent (blinded) assessments.

Results: After 3 months, the erectile function of men in the intervention group was significantly better than in the control group $(\mathrm{P}<0.001)$. Control patients who were given the intervention also significantly improved 3 months later $(\mathrm{P}<0.001)$. After 6 months, blind assessment showed that $40 \%$ of men had regained normal erectile function, $35.5 \%$ improved but $24.5 \%$ failed to improve.

Conclusion: This study suggests that pelvic floor exercises should be considered as a first-line approach for men seeking long-term resolution of their erectile dysfunction.

\section{Editorial Comment}

The first time that pelvic floor exercise was discussed and documented as a realistic alternative for treatment of erectile dysfunction was in 1993 by Claes \& Baert, for patients with mild degrees of venous leakage (1). The authors randomized a group of 150 consecutive male patients with erectile dysfunction and proven venous leakage to surgery of to a program of pelvic floor training. Surgery was not superior to the pelvic floor training program either subjectively or objectively. $42 \%$ of patients was satisfied with the program and refused surgery (1). Since then, a couple of works have been done on this subject, with somewhat good results $(2,3)$.

Doctor Grace Dorey and co-workers have been extensively working in this field, examining the role of pelvic floor muscle exercises (focusing on the bulbocavernosus and ischiocavernosus muscles) as a key to restoring erectile function. The present work is one more important contribution from this group. The authors concluded that pelvic floor muscle exercises should be considered as a first-line approach for erectile dysfunction. This might be more important in men seeking long-term resolution of erectile dysfunction without acute pharmacological and surgical interventions, which can cause significant side-effects.

\section{References}

1. Claes H, Baert L: Pelvic floor exercise versus surgery in the treatment of impotence. Br J Urol. 1993; 71: 52-7.

2. Ballard DJ: Treatment of erectile dysfunction: can pelvic muscle exercises improve sexual function?J Wound Ostomy Continence Nurs. 1997; 24: 255-64.

3. Van Kampen M, De Weerdt W, Claes H, Feys H, De Maeyer M, Van Poppel H: Treatment of erectile dysfunction by perineal exercise, electromyographic biofeedback, and electrical stimulation. Phys Ther. 2003; 83: 536-43.

Dr. Francisco J.B. Sampaio

Full-Professor and Chair, Urogenital Research Unit State University of Rio de Janeiro

Rio de Janeiro, Brazil 


\section{Apoptosis and proliferation in human undescended testes}

Ofordeme KG, Aslan AR, Nazir TM, Hayner-Buchan A, Kogan BA

The Urological Institute of Northeastern New York, and the Division of Urology, Department of Surgery, and Department of Pathology, Albany Medical College, Albany, NY, USA

BJU Int. 2005; 96: 634-8

Objective: To study apoptosis and proliferation in the testes of children with undescended testes; the degree to which undescended testes contributes to a patient's ultimate fertility is debatable, but undescended testes have fewer germ cells, and some have proposed that apoptosis is an important cause.

Patients and Methods: Testis biopsies were taken at the time of orchidopexy in a consecutive series of children undergoing surgical repair for undescended testes. Immunohistological techniques were used to detect apoptosis and proliferation, and the numbers of cells undergoing apoptosis or proliferation per 50 seminiferous tubules were recorded.

Results: Inguinal testes had less apoptosis than abdominal testes, with a mean (sd) of 0.71 (1.31) vs 1.63 (1.95) apoptotic cells per 50 seminiferous tubules $(\mathrm{P}<0.02)$. Similarly, there was less apoptosis in children aged $>1$ years than in children aged $<1$ years $(0.68(1.40)$ vs $1.35(1.56) ; \mathrm{P}<0.03)$. Proliferation was very limited in all cryptorchid testes. In contrast to cryptorchid testes, five autopsy controls had many more apoptotic cells, (10.60 (1.34) per 50 seminiferous tubules), and many more proliferating cells, (8.40 (6.43) per 50 seminiferous tubules).

Conclusion: In contrast to animal studies, neither apoptosis nor proliferation was common in undescended testes from 6 months of age onward. However, apoptosis was more common in abdominal testes and in children aged $<1$ year. It is likely that, if substantial apoptosis occurs in human undescended testes, it occurs before 6 months of age.

\section{Editorial Comment}

Apoptosis has been implicated in testicular germ cell loss in experimental models of cryptorchidism. Using the rat as an experimental model, it was demonstrated that apoptosis is the predominant mechanism of germ cell death rather than atrophy and necrosis in cryptorchidism $(1,2)$. The mechanisms of germ cell apoptosis have been associated with oxidative stress or testicular exposure to elevated temperature, and there are evidence that endothelial nitric oxide synthase plays a functional role in mouse spermatogenesis in cryptorchidisminduced apoptosis (3).

In the present elegant and well designed study, doctor Kogan and his group, by the first time, showed us that surprisingly and different from animal studies, neither apoptosis nor proliferation was common in undescended testes from children with more than 6 months of age. The authors discussed that this unexpected result is probably due to the timing of the biopsies, as significant apoptosis might have taken place before the typical time of surgical intervention (6 months).

\section{References}

1. Kocak I, Dundar M, Hekimgil M, Okyay P: Assessment of germ cell apoptosis in cryptorchid rats. Asian J Androl. 2002; 4: 183-6.

2. DeFoor WR, Kuan CY, Pinkerton M, Sheldon CA, Lewis AG: Modulation of germ cell apoptosis with a nitric oxide synthase inhibitor in a murine model of congenital cryptorchidism. J Urol. 2004; 172(4 Pt 2): 1731-5; discussion 1735.

3. Ishikawa T, Kondo Y, Goda K, Fujisawa M: Overexpression of endothelial nitric oxide synthase in transgenic mice accelerates testicular germ cell apoptosis induced by experimental cryptorchidism. J Androl. 2005; 26: 281-8.

Dr. Francisco J.B. Sampaio

Full-Professor and Chair, Urogenital Research Unit State University of Rio de Janeiro Rio de Janeiro, Brazil 